\title{
ON NON-MEASURABLE SETS OF POINTS, WITH AN EXAMPLE*
}

BY

EDWARD B. VAN VLECK

LEBESGUE's theory of integration is based on the notion of the measure of a set of points, a notion introduced by BoREL and subsequently refined by LEBEsGUE himself. The question whether there are non-measurable sets of points was taken up by LEBEsGUE $\nmid$ a few months ago in a memoir bearing the title Contribution a l'étude des correspondances de M. Zermelo. While yet unaware that the question of their existence was here considered, I constructed recently in quite a different manner a very simple example of a non-measurable set, an example which has some especial interest when considered after LEBEsGUE in the light of correspondences. To give this example is the object of $\S 1$. It is obtained by splitting up the unit interval into a countable set of points and two superimposable sets of points of upper measure 1. Each of these in turn can be split into two non-measurable sets $(\$ 2)$, also of upper measure 1 , and so on.

$\S 2$ gives a brief, general analysis of non-measurable sets of points, which so far as I know is entirely new. It is shown that when any two complementary non-measurable linear point-sets are resolved each into two components, one of which is a subset of maximum measure $\S$ and the other of which is not measurable, the sum of the two non-measurable components is measurable and its measure is equal to the upper measure of either component.

Finally, the intimate connection between the upper and lower measures of any linear set is revealed by showing that not only is the lower measure equal to the measure of a subset of maximum measure, but the difference between the upper and lower measures is equal to the upper measure of the residual non-measurable component (Theorem 2).

\section{§1. Example of a non-measurable set of points.}

Let $S$ denote any set of points within the interval $(0,1)$, and let $C$ be the complementary set. The upper measure of $S$-call it $\bar{m}(S)$ - is the lower

\footnotetext{
* Presented to the Society February 29, 1908. Received for publication March 7, 1908.

† LFBragre, Bulletin de la societé mathematique de Fiance, vol. 35 (1907), p. 212.

$\$$ Not neceasarily unique.
} 
limit to the sum of a countable set of intervals inclosing $S$ in their interior. If in the set a number of intervals successively overlap, they can be combined into a single one. Hence a set of non-overlapping intervals may be used, the extremities of which are points of $C$. To inclose a point set we shall use henceforth only sets of intervals whose terminal points belong to the complementary point set.

Suppose now that $\bar{m}(S)<1$. Then $S$ can be inclosed in a set of non-overlapping intervals whose sum $1-\Sigma$ is as nearly equal to $\bar{m}(S)$ as we please, and the points exterior to the interior of these intervals form a closed subset of $C$ having a measure equal to $\Sigma$.

I shall now restrict $S$ and $C$ by the following condition: In $(a, b)$, any subinterval whatsoever of $(0,1)$, there can be found an interval as nearly equal in length to $a b$ as we please and such that the subsets of $C$ and $S$ included in it shall be similar to $C$ and $S$. In other words, $C$ and $S$ can be obtained from their subsets in this portion of $a b$ by mere magnification. In place of a single portion of $a b$ having the property postulated it will serve my purpose as well if there is a finite number of such portions, the sum of whose lengths differs from $a b$ by as little as we please. This condition secures for $C$ and $S$ what I shall term a homogeneous character. A familiar instance will be obtained by selecting for $C$ the rational points in $(0,1)$ or the points having the abscissas $m / 2^{n}$, in which $m$ and $n$ are arbitrary positive integers.

Under this restriction it can now be affirmed that either $S$ and $C$ are measurable and one of them has the measure 0 , or they are both non-measurable and have an upper measure equal to 1.

To prove this, suppose first that $\bar{m}(S)<1$. Inclose $S$ in a first set of intervals of measure $1-\Sigma<1$. Then the points exterior to their interior form a first subset $C^{(1)}$ of $C$ having a measure $\Sigma$. Now in each interval of this first set of intervals we can find by hypothesis a subinterval (or a finite number of subintervals with a total length) as nearly equal to it in length as we please and such that the subset of $C$ which it contains is similar to $C$. Consequently in this subinterval (or in each of the finite number of subintervals) there can be selected a set of intervals which is similar to the "first set" and which has its end points in $C$. The points of the subinterval (or subintervals) exterior to the interior of this set of intervals form a subset $C^{\prime}$ of $C$ which is similar to $C^{(1)}$ and exterior to $C^{(1)}$. If every interval of the first set of intervals is treated in this way we obtain a second set of intervals entirely within the first, and the measure of this second set may be taken greater than $(1-\epsilon)(1-\Sigma)^{2}$, where $\epsilon$ denotes a small arbitrarily prescribed positive quantity. The subsets $C^{\prime}$. in the first set of intervals which are excluded from the second set form, when taken together, a second subset $C^{(2)}$ of $C$ which has a measure greater than $(1-\epsilon)(1-\Sigma) \Sigma$. Let the second set of intervals be treated in the same manner as the first, and 
so on. Continuing in this manner we find that $C$ contains distinct subsets, the measures of which are greater than the successive terms of the progression

$$
\Sigma+(1-\epsilon)(1-\Sigma) \Sigma+(1-\epsilon)^{2}(1-\Sigma)^{2} \Sigma+\cdots=\frac{\Sigma}{\Sigma+\epsilon(1-\Sigma)} .
$$

As $\epsilon$ is arbitrary, it follows that $C$ has a component with a measure as nearly equal to 1 as we please. Taking for $\epsilon$ a sequence of values approaching 0 as their limit, we obtain measurable components of $C$ whose sum has a measure equal to 1 . The set of points in $(0,1)$ complementary to this sum has the measure 0 and consists of the remaining points of $C$ and all of the points of $S$. Therefore we conclude that if $\bar{m}(S)<1$, the sets $C$ and $S$ are measurable, and the latter has the measure 0 .

If, on the other hand, $\bar{m}(C)<1$, we may adjoin to $S$ the two terminal points 0,1 and repeat the reasoning with the interchange of $S$ and $C$. In this repetition certain points in the $i$ successive sets of intervals $(i=1,2,3, \ldots)$, which correspond to the terminal points 0,1 of the initial set, must either be neglected or be adjoined to $S$. This, however, is absolutely without influence upon the reasoning, as these points are countable and the measure of a countable set of points is 0 .

Lastly, if $\bar{m}(S)=\bar{m}(C)=1$, neither set can be measurable since the sum of their upper measures exceeds the length of the unit-interval. We thus reach the conclusion announced that either $S$ and $C$ are measurable and the measure of one of them is 0 , or they are both non-measurable with an upper measure equal to the length of the interval.

We are now ready to begin the construction of two non-measurable sets $S$ and $C$. For this purpose divide the unit-interval arbitrarily into pairs of points, $x$ and $y=1-x$, which are the images of each other with respect to the middle point of the interval. The middle point can be neglected or added to $S$ or $C$. Any two point sets $\{x\}$ and $\{y\}$ thus constructed must have equal upper measures, as either set, regarded as a rigid system, can be superimposed by reflection upon the other. If they are measurable, their common upper measure is $\frac{1}{2}$. To prevent this I shall secure for $S$ and $C$ the "homogeneous character" previously discussed.

To this end we will associate with each $x$ in the set $S$ the countable set $\left\{x \pm n / 2^{n}\right\}$ and correspondingly with each $y$ in $C$ the set of points $\left\{1-\left(x \pm m / 2^{n}\right)\right\}$, in which $m$ and $n$ denote arbitrary positive integers. These two sets of points are entirely distinct unless $x$ itself and hence all points of both sets have the form $m^{\prime} / 2^{n^{\prime}}$. Points with abscissas of this special form may either be assigned arbitrarily to $S$ and $C$ or neglected. Instead of so doing, however, we shall put aside these points into a separate point set $K$. This, being countable, has the measure 0 and its removal will in no wise affect $\bar{m}(S)$ or $\bar{m}(C)$. 
The restriction just made upon the assignment of the points of the unit interval to $S$ and $C$ secures for these two point sets the following property. If the interval is divided into $2^{n}$ equal parts each of these parts, in respect to the distribution of the points of $S$ and of $C$, is an exact copy of every other part. Consequently, to secure their similarity to the initial unit interval, $I$ have merely to require, if possible, that whenever a point $x$ is assigned to $S$ the points $x / 2, x / 4, x / 8, \ldots$ shall be assigned to the same set, and conversely, $x$ shall be assigned simultaneously with any $x / 2^{n}$. This requirement will give rise to a conflict in the distribution of the points of $(0,1)$ to $S$ and $C$ if, and only if, a point $x / 2^{p^{\prime}} \pm m^{\prime} / 2^{n^{\prime}}$ should coincide with one having the coördinate $1-\left(x / 2^{p^{\prime \prime}} \pm m^{\prime \prime} / 2^{n^{\prime \prime}}\right)$, in which $p^{\prime}, m^{\prime}, n^{\prime}, p^{\prime \prime}, m^{\prime \prime}, n^{\prime \prime}$ denote non-negative integers. But clearly this can occur only when $x$ is rational. Let all the rational points of the unit interval be thrown into our negligible set $K$ of measure 0 . After the rational points are thus set aside, the points of the interval may be separated into pairs of sets of countable points,

$$
\left\{\frac{x}{2^{ \pm p}} \pm \frac{m}{2^{n}}\right\}, \quad\left\{1-\left(\frac{x}{2^{ \pm p}} \pm \frac{m}{2^{n}}\right)\right\} \quad(m, n, p=0,1,2, \cdots),
$$

to be assigned respectively to $S$ and $C$.

The homogeneous structure desired has now been obtained. Furthermore, when the unit interval is divided into any $2^{n}$ equal parts, these parts are copies of one another in respect to the distribution of points of $K$ as well as $S$ and $C$, and are similar to the whole interval. Consider now the two complementary sets $S$ and $C+K$. If any subinterval $(a, b)$ of $(0,1)$ is taken, two points $a^{\prime}=m_{1} / 2^{n}$ and $b^{\prime}=m_{2} / 2^{n}$ may be found in the interior so near to $a$ and $b$ respectively that $a b-a^{\prime} b^{\prime}$ is as small as we please, and $a^{\prime} b^{\prime}$ consists of $m_{2}-m_{1}$ equal portions of length $1 / 2^{n}$ which are similar to the initial unit interval in respect to the distribution of the points of $S$ and $C+K$. Since these two sets are of equal upper measure, it follows from the preceding discussion that they are non-measurable and have an upper measure 1. By subtraction of $K$ from $C+K$ the same is seen to hold for $C$.

In dividing thus the unit interval into two non-measurable sets the separation has been left indeterminate, in so far as it was permitted to assign arbitrarily one of two image points $x$ and $1-x$ to $S$, each of the two points determining then one of the groups (1). To remove the arbitrary element of choice it is not at all necessary to give a law which will pick out uniquely a principal element from each group $\left(x / 2^{ \pm p} \pm m / 2^{n}\right)$. This appears, in consequence of a remark of LEBesGue,* to be impossible. Rather, the removal of the arbitrary feature depends on our ability to give a law which, out of an uncountable infinity of pairs of groups, will select one of each pair. Suppose now that we express any

* Bee LeBesgur's footnote, loa. oit., p. 210. 
point $x$ in the binary system; thus

Then

$$
x=. \alpha_{1} \alpha_{2} \alpha_{3} \cdots \quad\left(\alpha_{i}=0 \text { or } 1\right) .
$$

$$
1-x=\beta_{1} \beta_{2} \beta_{3} \cdots \quad\left(\alpha_{1}+\beta_{1}=1\right) .
$$

Any other point $x / 2^{ \pm p} \pm m / 2^{n}$ in the same group as $x$ will agree with $x$ in the succession of digits 0 and 1 after the omission of a finite number of initial elements, $\alpha_{1}^{\prime}, \alpha_{2}^{\prime}, \cdots, \alpha_{k}^{\prime}$ and $\alpha_{1}, \cdots, \alpha_{j}$, in the two numbers compared. The problem, therefore, which presents itself is to make a choice between $x=._{1} \alpha_{2} \ldots$ and $1-x=\beta_{1} \beta_{2} \ldots$ based on the ultimate form of the binary decimal and not at all on the initial elements. Such a choice can be made, if not for the continuum $(0,1)$, at least for many subsets. For example, suppose the ratio of the number of zeros to the number of 1 's in $\alpha_{1} \alpha_{2} \cdots \alpha_{n}$ to have as the abscissas of its largest and smallest points of condensation, for increasing $n$, the values $A$ and $B$. Then the corresponding values for $1-x$ are $1 / B$ and $1 / A$. Unless $A=1 / B$ it is clearly possible to give a rule for selecting one point from each pair, $x$ and $1-x$. Take now in the unit-interval the set which consists of all points for which $A \neq 1 / B$. This has a homogeneous character and is its own image with respect to $x=\frac{1}{2}$. If its upper measure is not 0 , it may replace the unit-interval in our previous reasoning, and can be split up into two non-measurable superimposable subsets in precisely the same way as the unit interval. Thus it seems to me possible, and perhaps not difficult, to remove the arbitrary element of choice in my example by confining one's attention to a proper subset of the continuum, though as yet I have not succeeded in proving that this is possible.

It remains to ascertain to what categories the sets $S$ and $K$ belong. 'By BaIre's definition a point set of the first category is one that can be created by putting together a countable number of sets, each of which is nowhere dense. Now $S$ and $C$ are superimposable, and this is true also for their subsets included in any portion $a b$ of our unit interval. This shows that they are throughout of the second category,* for otherwise some subinterval of the unit interval would be obtained as the sum of point sets, $S, C$, and $K$ of the first category, but this is impossible.

\section{§2. On the theory of non-measurable sets.}

In the preceding section an interval has been split up into two complementary non-measurable sets, each of which has an upper measure equal to the measure of the sum. That a similar decomposition always takes place whenever a measurable linear set is resolved into two non-measurable sets will appear from the following analysis.

* I use here the term second category to denote a set which is not of the first category, rather than in the sense of BAIRE. C. LeBbraUt, 1. 0., p. 212. 
Take for consideration any two complementary non-measurable sets $S$ and $C$ in the unit interval. If $\bar{m}(S)$ and $\bar{m}(C)$ are not both equal to 1 , suppose $\bar{m}(S)<1$, and adjoin to $C$, if necessary, the points $x=0, x=1$. Then $S$ may be so inclosed within a set of intervals having their end points in $C$ that the closed sets of points exterior to the interior of these intervals shall have a measure differing from $1-\bar{m}(S)$ by less than a small, arbitrarily assigned, positive $\epsilon$. Taking for $\epsilon$ a sequence of values having 0 as its limit we obtain a countable set of measurable closed subsets of $C$. The sum of these subsets has a measure not less than $1-\bar{m}\left(S^{r}\right)$. Clearly also its measure can not exceed this amount, for otherwise the measure of the complementary set would be less than $\bar{m}(S)$, and hence also the upper measure of $S$ which is included in it. This shows that $C$ contains a subset $C_{1}$ of maximum measure $1-\bar{m}\left(S^{\prime}\right)$. Similarly $S$ has a subset $S_{1}$ of maximum measure $1-\bar{m}(C)$.

Place now

$$
S=S_{1}+S^{\prime}, \quad C=C_{1}+C^{\prime}
$$

Together $S^{\prime}$ and $C^{\prime}$ make a measurable set $T$ such that

But

and

$$
m\left(S_{1}\right)+m\left(C_{1}\right)+m(T)=1
$$

$$
\begin{aligned}
& 1=\bar{m}(S)+m\left(C_{1}\right), \quad 1=\bar{m}(C)+m\left(S_{1}\right), \\
& \bar{m}(S) \leqq m\left(S_{1}\right)+\bar{m}\left(S^{\prime}\right), \quad \bar{m}(C) \leqq m\left(C_{1}\right)+\bar{m}\left(C^{\prime}\right)
\end{aligned}
$$

Multiplying equation (2) by 2 and then adding to them (3) and (4), we find that

$$
2 m(T) \leqq \bar{m}\left(S^{\prime}\right)+\bar{m}\left(C^{\prime}\right) .
$$

Since $S^{\prime}$ and $C^{\prime}$ are subsets of $T$, this can be only if

$$
\bar{m}\left(S^{\prime}\right)=\bar{m}\left(C^{\prime}\right)=m(T) .
$$

The analysis and result hold also when we assume $\bar{m}(S)=1$, also if we split up $S$ and $C$ in any other way, each into a subset of maximum measure (necessarily the same maximum) and a non-measurable subset. We reach therefore the following result:

TheOREM 1. If $S$ is any non-measurable linear aggregate and $C$ is its complement, let them each be resolved in any way into a subset of maximum measure and a non-measurable subset. Then the two non-measurable components taken together make a measurable set, and the upper measure of either component is equal to the measure of their sum.

It is to be noticed that the use necessarily of the sign of equality in (5) carries with it the use of the same sign in (4). Or in other words :

Theorem 2. For any non-measurable set $S$ we have

$$
\bar{m}(S)=m\left(S_{1}\right)+\bar{m}\left(S^{\prime}\right)
$$


in which $S_{1}$ denotes any subset of $S$ of maximum measure and $S^{\prime}$ is the residual non-measurable subset.

LEBESGUE's lower measure $\underline{m}(S)$ is by definition $1-\bar{m}(C)$ or, by virtue of the last theorem, $1-m\left(C_{1}\right)-\bar{m}\left(C^{\prime}\right)$. Since also $\bar{m}\left(C^{\prime}\right)=m(T)$, we see from equation (2) that the lover measure $\underline{m}(S)$ of any linear point set $S$ is equal to the measure of the subset $m\left(S_{1}\right)$ of maximum measure which it contains. This is equivalent to W. H. Young's definition of the inner content* of a set as " the upper limit of the content of its closed components."

Theorems 1 and 2 seem to me to advance considerably the theory of measure and of non-measurable point sets. In particular, theorem 2 throws new light on the connection between the upper and lower measures. Young's definitions of the two measures, unlike LeBEsGue's, were of very diverse nature. Equation (2) shows that he, nevertheless, was concerning himself with the essential nature of a point-set. By treating the residue $S^{\prime}$, neglected by Young, I have brought out the close connection between the definitions of Young, and the development has been simple and rapid, connecting closely with both LEBEsGUE and Young.

Several corollaries $\dagger$ can be developed rapidly from theorams 1 and 2. For example, it follows at once that the upper measure of the sum of a measurable and non-measurable set which have no point in common is the sum of their uppes measures.

I scarcely need to remark that any subset of the non-measurable component $S^{\prime}$ is likewise non-measurable, unless, of course, it is of measure 0 .

The inference should not be hastily made on the basis of theorem 1 that a new and more comprehensive definition of measure is possible; to wit, as the measure of the maximum measurable component plus one-half of the irreducible residue. For such a definition it is not true, in general, that the measure of the sum of two sets without a common point is the sum of their measures. To see this it will suffice to show that just as the unit interval was resolved into two subsets of upper measure 1 , so either of these can be resolved into turn into two subsets of upper measure 1 , and so on ad infinitum.

To effect such a resolution return to the non-measurable set $S$ of $\S 1$. In this we put together groups of points having the abscissas $x \pm m / 2^{n}$. For a fixed value of $x$ and of $n$ a series of $2^{n}$ equidistant points is obtained. These we will now separate into two groups of $2^{n-1}$ points in the following manner. If, for convenience, the points are denoted in the order of increasing abscissa by $x_{1}, x_{2}, \ldots, x_{2^{n}}$, let the first two points be assigned one to one group and one to the other; let the next two be assigned in the opposite way so that $x_{1}$ and $x_{3}$ do

* Young's Theory of Point Sets, p. 96. His definition is reconciled with LmBEsaun's definition on p. 106.

† See, in partionlar, the latter part of Young's Chap. 5. 
not belong to the same group, nor $x_{2}$ and $x_{4}$; the next four points in a manner opposite to that of assigning the first four so that $x_{i+4}$ and $x_{i}(i=1,2,3,4)$ belong to different groups ; the next eight so that $x_{i+8}$ and $x_{i}(i=1,2, \ldots, 8)$ do not fall in the same group; and so on. Now $x$ itself, being one of the $2^{n}$ points $x_{i}$, falls in one or the other of our two groups. According as the group does or does not contain $x$, we will assign it to one or the other of two components $S^{\prime}, S^{\prime \prime}$ into which $S$ is to be split. It is seen very easily that this soparation of the points $\left\{x \pm m / 2^{n}\right\}$ into two groups for fixed $x$ and for two consecutive values of $n$ is consistent the one with the other; in fact, the assignment of the points $\left\{x \pm m^{\prime} / 2^{n+1}\right\}$ is completely determined by the previous assignment of the points of its subset $\left\{x \pm m / 2^{n}\right\}$.

Let this separation be effected first for any fixed $x$ belonging to $S$ and for all values of $n$. Finally, by varying $x$ within $S$ we effect a decomposition of $S$ into two components $S^{\prime}, S^{\prime \prime}$. These two components must be of equal upper measure, for by construction $x$ and $x \pm \frac{1}{2}$ belong the one to $S^{\prime}$ and the other to $S^{\prime \prime}$. Thus the upper measure of the subset of $S^{\prime}$ or of $S^{\prime \prime}$ in the interval $\left(0, \frac{1}{2}\right)$ is the same as the upper measure of $S^{\prime \prime}$ or of $S^{\prime}$ respectively in $\left(\frac{1}{2}, 1\right)$.

To secure a quasi-homogeneous structure for $S^{\prime}$ and $S^{\prime \prime}$ we will add the requirement that the entire sequence of points $x, x / 2, x / 4, x / 8, \ldots$ shall belong to $S^{\prime}$ (so also to $S^{\prime \prime}$ ) whenever any one point $x / 2^{n}$ of the sequence so belongs. This insures that the subsets of $S^{\prime}, S^{\prime \prime}$ in $\left(0,1 / 2^{n}\right)$ shall be similar in distribution to the whole sets $S^{\prime}, S^{\prime \prime}$ in the unit interval. Furthermore, from our mode of separating the points $\left(x \pm m / 2^{n}\right)$ for fixed $n$ and $x$ into two groups, it follows that if the unit interval is divided into $2^{n}$ equal parts, $2^{n-1}$ of these parts will be identical in respect to distribution of the subsets of $S^{\prime}$ and $S^{\prime \prime}$ which they contain and will be similar therefore to the unit interval, as well es the first half of the same. The other $2^{n-1}$ parts are also identical and are obtained from the preceding parts by the interchange of the points of $S^{\prime}$ and those of $S^{\prime \prime}$, being similar to the second half of the unit interval. Consider now any portion $a b$ of the unit interval. Two points $a^{\prime}=m_{1} / 2^{n}, b^{\prime}=m_{2} / 2^{n}$ in the interior may be sclected as near to $a$ and $b$ as we please, and the intervening intervals consist of $2\left(m_{1}-m_{2}\right)$ equal parts of length $1 / 2^{n+1}$. The half of this number are similar to the first half of unit interval, and the other $m_{1}-m_{2}$ parts are similar to the second half of the unit interval. The argument of $\S 1$ may therefore be applied with immaterial change to the complementary sets $S^{\prime}$ and $S^{\prime \prime}+C+K$. Because $S^{\prime}$ and $S^{\prime \prime}$ have by construction equal upper measures at least as great as $\frac{1}{2}$, it follows from $\S 1$ that $S^{\prime}$ and hence $S^{\prime \prime}$ is not measurable, and that their common upper measure is 1 .

Slight reflection will make it apparent that $S^{\prime}, S^{\prime \prime}$ can be resolved on similar principles each into two components of upper measure 1 , and so on indefinitely.

UNIVERgITY OF WIsconsin, MaDison, WIs. 